\title{
Médiévales
}

Langues, Textes, Histoire

\section{Approche interdisciplinaire d'un bois méditerranéen entre la fin de l'antiquité et la fin du Moyen Âge, Saugras et Aniane, Valène et Montpellier}

An interdisciplinary approach of a Mediterranean wood between the end of Antiquity and the end of the Middle Ages.

Charlotte Britton, Lucie Chabal, Gaspard Pagès et Laurent Schneider

\section{OpenEdition}

Journals

Édition électronique

URL : https://journals.openedition.org/medievales/3573

DOI : $10.4000 /$ medievales.3573

ISSN : 1777-5892

Éditeur

Presses universitaires de Vincennes

Édition imprimée

Date de publication : 1 décembre 2007

Pagination : 65-80

ISBN : 978-2-84292-211-5

ISSN : 0751-2708

Référence électronique

Charlotte Britton, Lucie Chabal, Gaspard Pagès et Laurent Schneider, « Approche interdisciplinaire d'un bois méditerranéen entre la fin de l'antiquité et la fin du Moyen Âge, Saugras et Aniane, Valène et Montpellier », Médiévales [En ligne], 53 | automne 2007, mis en ligne le 17 décembre 2009, consulté le 24 avril 2022. URL : http://journals.openedition.org/medievales/3573 ; DOI : https://doi.org/10.4000/ medievales.3573 
Charlotte BRITTON

Lucie CHABAL

Gaspard PAGÈS

Laurent SCHNEIDER

\section{APPROCHE INTERDISCIPLINAIRE \\ D'UN BOIS MÉDITERRANÉEN ENTRE LA FIN DE L'ANTIQUITÉ \\ ET LA FIN DU MOYEN ÂGE : SAUGRAS ET ANIANE, VALÈNE ET MONTPELLIER}

Depuis 1999, une Action Thématique Incitative sur Programme du département SHS du Cnrs, relayée en région par le soutien du Service Régional de l'Archéologie (MCC), a pour objet d'étude un espace de garrigue languedocienne situé à une quinzaine de kilomètres au nord-ouest de Montpellier. Ce projet est né de la volonté de porter une attention plus soutenue à des espaces dits de marges et à une typologie particulière d'établissements trop vite considérée par l'historiographie comme de sommaires « habitats refuges » mis en place à la fin de l'Antiquité dans le contexte événementiel des « invasions barbares ». L'approche s'inscrit dans la longue durée, de l'Antiquité au seuil de l'époque moderne et dans des jeux d'échelles entre sites archéologiques, micro-région et horizon urbain. Elle se nourrit aussi bien d'investigations archéologiques de terrain (fouilles, sondages, prospections) que d'études d'archives (fonds consulaire de Montpellier) et d'analyses de laboratoire (anthracologie, carpologie, paléométallurgie...). Le thème de ce numéro de Médiévales nous permet aujourd'hui de présenter quelques résultats de ce projet collectif en insistant sur les ressources forestières d'un espace de garrigue méditerranéenne encore trop souvent pensé comme exclusivement voué au pastoralisme.

Des siècles obscurs ? Résilience de l'occupation humaine et précocité d'une économie forestière et artisanale $\left(\mathrm{V}^{\mathrm{e}}-\mathrm{IX} \mathrm{e}^{\mathrm{e}}\right.$ siècles $)$

Les petits causses des garrigues montpelliéraines entrent dans l'histoire à partir des dernières années du VIII ${ }^{\mathrm{e}}$ siècle. La documentation écrite la plus ancienne y place les premiers noyaux patrimoniaux qui sont associés à la 
fondation du monastère d'Aniane par le fils d'un aristocrate wisigoth, Witiza. Plus connu sous le nom de saint Benoît d'Aniane, le personnage devait faire de son sanctuaire, plus d'un siècle avant Cluny, l'un des fers de lance d'une réforme monastique appelée à gagner tout l'Empire sous le règne de Louis le Pieux. Établi au débouché des gorges de l'Hérault, le monastère d'Aniane disposa dès sa fondation de deux complexes fonciers principaux. L'un, situé de part et d'autre de la Mosson, à proximité de la voie domitienne et de la colline de Montpellier était constitué à partir d'un ancien fisc royal (Juvignac) et géré par une cella nova (quartier de Celleneuve dans l'agglomération actuelle de Montpellier). Le second, Saugras, situé peu ou prou à midistance entre Juvignac et le monastère d'Aniane, est plusieurs fois qualifié de locus. Les moines y avaient établi dès les dernières années du viII ${ }^{\mathrm{e}}$ siècle une deuxième cella qui constitua longtemps la tête de réseau de leur présence dans ce secteur. Depuis la première confirmation de ce patrimoine par le roi Charles en 799 jusqu'à Charles le Chauve en 852, différents diplômes rassemblés dans le cartulaire $\mathrm{du} \mathrm{XII}^{\mathrm{e}}$ siècle, énumèrent la liste de ces lieux demeurés, de fait, associés à l'histoire des origines du monastère ${ }^{1}$. L'absence de données archéologiques a longtemps laissé supposer que les moines de Benoît avaient joué un rôle pionnier dans la colonisation progressive de ces espaces de marges que l'on imaginait largement sous-peuplés et inexploités avant les $\mathrm{XI}^{\mathrm{e}}-\mathrm{XII}^{\mathrm{e}}$ siècles. Or, sur ce point, l'archéologie apporte aujourd'hui un tout autre éclairage. Dans le locus de Saugras, un vaste éperon calcaire, détaché par de hautes falaises, avait été occupé bien avant l'arrivée des moines, dès la seconde moitié $d u v^{\mathrm{e}}$ siècle. Sept ans de fouilles montrent désormais qu'il ne s'agissait pas là, loin s'en faut, d'un simple habitat refuge, concept qui masquait en fait l'absence de documentation.

\section{Marges montagnardes et sites refuges : des données à reconsidérer}

Le site du Roc de Pampelune (Argelliers, Hérault) apparaît aujourd'hui comme un pôle de peuplement structurant, un habitat de conquête qui prend la forme d'une véritable agglomération. À l'intérieur d'une enceinte maçonnée dotée d'ouvrages de flanquement, de nombreuses constructions, maisons et locaux techniques construits en pierre et couverts de tuiles, se déploient en quartiers agglomérés ou autour de petites cours sur une superficie de plus de deux hectares. Amphores orientales et surtout africaines, lampes et vaisselles importées montrent par ailleurs l'insertion quasi-immédiate de ces habitants dans les circuits du grand commerce méditerranéen et obligent à reconsidérer l'idée d'une installation autarcique. La présence d'un rempart témoigne par ailleurs d'une certaine cohésion communautaire de la population rassemblée, cohésion que relèvent encore d'autres équipements collectifs

1. L. SCHNEIDER, «À propos de l'espace rural durant le haut Moyen Âge méridional : archéologie et cartulaires » dans D. LE BLEVEC dir., Les cartulaires méridionaux, Actes du colloque de Béziers, 20-21 sept. 2002, Paris, 2006, p. 50-54. 
dont une église dotée d'un baptistère. Le plan de l'agglomération, l'homogénéité des techniques de construction, l'aspect modulaire de certaines maisons, la création ex nihilo de l'ensemble sont autant d'éléments qui suggèrent que la décision d'implanter une communauté humaine dans ce secteur a dû relever d'une action programmée. Initiative locale ou d'État, il est difficile de répondre, mais l'on doit noter que la forme adoptée par ce nouvel établissement (aggloméré, perché et fortifié) et le type d'espace qu'il contrôle (forestier) apparaissent en cette seconde moitié du v $v^{\mathrm{e}}$ siècle comme des traits innovants ou du moins opposables au mode d'exploitation «en villae » qui avait prévalu pendant tout l'Empire dans les zones de plaine et de piémont de la Narbonnaise. Bien que l'établissement soit fortifié, rien n'évoque de manière flagrante dans la culture matérielle une occupation de type militaire. Bien au contraire, les découvertes répétées d'outils (faux, faucilles, houes, haches, gouges...), de pesons de métier à tisser, de hangars et de bergeries témoignent de pratiques sylvo-agro-pastorales. Au fur et à mesure que les fouilles se sont développées est apparue cependant avec plus d'évidence encore la part importante des activités artisanales dans l'économie de la place. Les arts du feu sont particulièrement bien représentés. La métallurgie est sans doute l'activité la plus lisible dans la culture matérielle du fait de sa propension à produire des déchets. Les fouilles ont ainsi livré plus de cent kilogrammes de scories ferreuses, déchets directement issus des différentes phases de travail de la métallurgie du fer. Cette documentation a été traitée à l'aide de méthodes archéométriques appropriées. Les analyses métallographiques ont permis de déterminer que la seule phase de travail développée à l'intérieur de l'agglomération était celle du forgeage d'objets en fer à l'exclusion de toute autre pratique telle que la réduction du minerai. Le travail effectué par les forgerons du Roc de Pampelune témoigne par ailleurs de savoir-faire de grande qualité, comme l'attestent la précision et la finesse des traitements chimiques et mécaniques réalisés. Ce premier constat suggère que le mode de peuplement de cette agglomération nouvelle a été marqué par l'installation d'une main d'œuvre véritablement spécialisée. Concrètement, chacun des trois ateliers permanents repérés jusqu'ici répondait à des travaux différenciés.

L'un était tourné vers la production d'outils performants en acier tels que ciseaux à bois et à pierre, couteaux..., un autre vers la fabrication de petits objets en fer pur (mobiliers domestiques) et le dernier vers l'épuration et la mise en forme de demi-produits. Issus de l'épuration et du compactage de loupes produites par la réduction directe en bas fourneau du minerai de fer, ces demi-produits se présentent comme des masses d'alliage ferreux prêtes à être façonnées par le forgeron ${ }^{2}$.

2. G. Pagès, L. Schneider et P. Fluzin, «Le travail du fer dans l'établissement perché tardo-antique du Roc de Pampelune (Argelliers, Hérault) : l'apport des analyses métallographiques », ArchéoSciences, Revue d'Archéométrie, 29, 2005, p. 107-116 (http://halshs.ccsd.cnrs.fr/ halshs-00090912). 
L'analyse chimique (recherche des « inclusions-trace ») des demi-produits découverts sur le site indique quant à elle que les forgerons du Roc de Pampelune ont exercé leur activité à partir d'alliages ferreux majoritairement issus d'un lieu de production unique. De deux choses l'une : soit ce lieu de réduction se situait dans le proche périmètre de l'agglomération, car on a repéré différents gisements de minerai pisolithique, facilement extractible au pied même du site et plus largement dans un rayon de deux kilomètres, soit il se trouvait au contraire à moyenne ou grande distance, ce qui impliquerait des liens étroits et relativement constants entre cette entreprise et l'agglomération. La complexité de ce dernier schéma, et surtout notre ignorance actuelle de l'existence de grands ateliers de réduction tardo-antiques à l'échelle de la Narbonnaise Première, donnent finalement plus de poids à l'hypothèse d'un approvisionnement à petite distance ${ }^{3}$. Dans ce milieu pierreux, accidenté et boisé particulièrement rétif aux prospections de surface, la démonstration archéologique d'un tel scénario demeure cependant formellement difficile à établir. Toutefois la multiplication des investigations réalisées peut renforcer cette hypothèse par un biais parallèle. La population de l'agglomération du Roc de Pampelune comptait en effet d'autres types d'artisans. En sus de la métallurgie du fer, des personnels travaillaient non seulement les alliages cuivreux mais aussi le verre. L'artisanat du verre était jusqu'alors essentiellement repéré dans le midi de la France en milieu urbain (Arles, Marseille) ou autour de groupes épiscopaux (Maguelone). Dans l'agglomération qui nous occupe, l'identification d'un atelier apparaît donc comme un trait original, d'autant plus que la découverte de fragments de creusets dispersés en divers points montre que cette activité se déployait plus largement encore dans différents quartiers. Mais surtout, les prospections de surface ont permis d'identifier deux établissements spécialisés dans l'artisanat du verre, en dehors de l'enceinte, à moins de deux kilomètres de l'agglomération. Il faut donc envisager dans l'environnement forestier de l'établissement l'existence d'un réseau de sites secondaires contemporains qui participaient à l'économie de la place entre les $\mathrm{V}^{\mathrm{e}}$ et $\mathrm{VI}^{\mathrm{e}}$ siècles. L'activité était-elle organisée comme pour la métallurgie du fer en termes de chaînes opératoires sectorisant les phases de travail : réduction des minerais au plus près des matières premières dans les massifs forestiers, puis stockage et transformations des loupes en demiproduit ou en objets manufacturés au sein de l'enceinte ? Ce schéma est-il transférable pour l'organisation du travail du verre ? Faut-il distinguer une production primaire de lingots dans le massif forestier puis un stockage et une fabrique d'objets à l'intérieur de l'enceinte, ou au contraire envisager l'existence d'ateliers multiples dispersés en fonction de l'accès aux ressources et notamment aux combustibles ? Il est trop tôt pour répondre, mais il est désormais manifeste que la principale ressource naturelle recherchée, nécessaire à tous ces artisanats, était celle du bois et du charbon de bois.

3. G. PAGÈs, La métallurgie du fer en France méditerranéenne de l'Antiquité au début du Moyen Âge : jalons pour une approche pluridisciplinaire, Doctorat en cours, Université Paul Valéry-Montpellier III. 


\section{L'économie des combustibles : apport des analyses anthracologiques}

L'analyse des activités artisanales de l'agglomération peut également s'appuyer sur le combustible, à partir de l'échantillonnage systématique réalisé au moment des fouilles. Les acquis méthodologiques de l'anthracologie ${ }^{4}$ et les comparaisons spatiales et fonctionnelles au sein du site étudié permettent de raisonner, non seulement sur les usages du bois (activités domestiques et artisanales, construction) mais aussi sur les espaces d'approvisionnement par la composition des boisements reflétés. Les premières identifications de charbons de bois révèlent ainsi un usage d'essences très diversifié, provenant principalement d'une chênaie méditerranéenne assurément locale, faite de taillis, mais aussi d'arbres ayant donné des fûts. Le premier constat, comme pour d'autres sites, est que les habitants ont tiré profit au mieux de la végétation dans un environnement de proximité, exploitant de façon très complète les espèces arborescentes d'une chênaie largement traitée en taillis (Chêne blanc, Chêne vert, Érable de Montpellier) mais aussi les moindres arbustes qui en constituent le cortège habituel (Arbousier, Buis, Filaire, Bruyère, Genévrier, Pistachier...). Plus ponctuellement, on trouve des essences qui n'existent pas (ou plus) dans l'environnement actuel du site, comme le Pin noir de Salzmann (spontané en Languedoc, qui forme un boisement relique à St-Guilhem-le-Désert) ou, dans la charpente au côté du Chêne blanc, le Sapin et le Hêtre qui ont pu jusqu'à des périodes historiques exister à plus basse altitude qu'actuellement en zone méditerranéenne, même sans envisager de variation climatique ${ }^{5}$. Quoi qu'il en soit des distances parcourues pour acquérir ces espèces particulières, la majorité des ligneux utilisés témoigne d'une chênaie mature, riche en Chêne blanc, assez proche finalement de ce qu'elle était au Chalcolithique.

Mais qu'en est-il de chaque activité consommatrice de bois? Qu'il s'agisse de métallurgistes, de verriers ou de potiers (tuiliers), chacune de ces activités a ses propres exigences dans la conduite du feu qui fait l'objet de savoir-faire très précis. Pour la métallurgie, l'activité de forge révèle un éventail d'essences diversifié, riche en Chêne. On sait que le travail du fer nécessite impérativement l'emploi, comme combustible, de charbon de bois (ou de houille) qui seul peut fournir, dans la forge équipée d'un soufflet, une température élevée (supérieure à $1000^{\circ} \mathrm{C}$ ) sous la forme d'une chaleur radiative et conductive sans flamme et, à l'occasion, une atmosphère réductrice nécessaire à certaines phases de forgeage. L'anthracologie ne peut pas distinguer les résidus d'une combustion de bois de ceux d'une combustion de charbon de bois. Mais le contexte archéologique permet d'identifier l'activité et, par là, la nature du combustible. La question des essences utilisées rebondit alors sur celle des pratiques de charbonnage. On sait par les études

4. L. Chabal, Forêts et sociétés en Languedoc (Néolithique final, Antiquité tardive): l'anthracologie, méthode et paléoécologie, Paris, 1997 (Documents d'Archéologie Française, 63).

5. Ibid. 
anthracologiques de charbonnières en meules qu'à partir $\mathrm{du} \mathrm{XI}^{\mathrm{e}}$ siècle au moins en Languedoc, toutes les essences de taillis présentes autour de la faulde sont charbonnées et reflètent alors la composition des boisements ; des siècles de charbonnage intensif, au côté des autres coupes de bois, n'ont pas compromis la vitalité des taillis, mais ont conduit à un enrichissement relatif en Chêne vert dont ont hérité les paysages actuels ${ }^{6}$. Antérieurement, on connait mal le mode de charbonnage qui pouvait être en fosse. Qu'un grand nombre d'essences soit utilisé dans les forges de Pampelune n'est ainsi pas surprenant mais que la composition en essences soit proche du combustible de l'activité domestique est remarquable et permet d'envisager que le charbonnage nécessaire à l'activité a été pratiqué dans l'environnement immédiat du site, comme l'approvisionnement en bois de feu domestique. En effet, un achat de charbon de bois extérieur à ce périmètre du site aurait probablement révélé une composition différente. Il s'agit là d'informations précieuses, qui tendent à confirmer le caractère autonome du site pour une part importante de son activité économique, avec toutes les conséquences sociétales qui en découlent.

\section{Nouvelles conditions de production et d'organisation de l'habitat:} l'empreinte des années 450-550

L'agglomération du Roc de Pampelune, pôle de peuplement nouveau, implanté dans un secteur jusqu'alors peu pénétré par l'homme, regroupait une main d'œuvre compétente, sinon savante, spécialisée dans les arts du feu, notamment dans les artisanats du fer, des alliages cuivreux et du verre. Cette orientation économique impliquait parallèlement des pratiques locales de charbonnage, de bûcheronnage et peut-être plus généralement de travail et de commerce du bois. Loin de l'image connotée d'habitat refuge, trop vite assignée à ces établissements nés dans le contexte de l'émergence des royaumes barbares, cette catégorie d'établissements témoigne au contraire de réelles capacités d'investissement et d'organisation de la production en regroupant des personnels spécialisés à l'extérieur des villes et des agglomérations traditionnelles, dans des espaces ruraux qui offraient des ressources naturelles particulières, notamment forestières. Cela n'exclut évidemment pas des pratiques agro-pastorales et une certaine polyvalence des activités, mais cela rappelle, si besoin était, que le monde rural ne saurait être réduit à sa seule dimension agricole. Le village du Roc de Pampelune adopte d'ailleurs par bien des aspects un profil économique de type urbain qui révèle aussi, durant le premier tiers du $\mathrm{VI}^{\mathrm{e}}$ siècle la persistance et plus encore le renouvellement de réseaux d'échanges fiables, aspect que l'on perçoit également dans la culture matérielle, principalement à travers les céramiques et les amphores importées.

6. L. FABRE, Le charbonnage historique de la chênaie de Quercus ilex L. (Languedoc, France) : conséquences écologiques, Thèse de Doctorat, Université de Montpellier II, 1996. 
Il n'est pas lieu ici de discuter du statut politique de ces établissements «oppida, castra ou castella» du premier Moyen Âge dont une partie du maillage survit encore à l'époque carolingienne sous la forme de chefs-lieux de territoire ${ }^{7}$. Celui du Roc de Pampelune ne connut pour sa part qu'un siècle d'existence à peine. Dans le second quart $\mathrm{du}_{\mathrm{VI}}^{\mathrm{e}}$ siècle, un épisode encore difficile à interpréter (un incendie) a brutalement déstructuré l'agglomération et engagé l'abandon des lieux. Au seuil du $\mathrm{VII}^{\mathrm{e}}$ siècle, l'enceinte paraît entièrement désertée. Il faut attendre la fin du vIII ${ }^{\mathrm{e}}$ siècle pour qu'un nouveau noyau de population, bien plus modeste, soit installé, cette fois-ci à l'initiative des moines de Benoît d'Aniane, dans le vallon que dominait jadis l'agglomération. On ne sait quasiment rien de l'activité économique, supposée par défaut extensive, de ce petit pôle monastique. Tout au plus doit-on noter que le choix de l'implantation s'est effectué à quelques centaines de mètres seulement des ruines de l'agglomération $\mathrm{du}_{\mathrm{VI}}^{\mathrm{e}}$ siècle et que les fouilles témoignent d'une activité de récupération de matériaux. Si la portée économique d'une telle activité nous échappe, on doit néanmoins pouvoir avancer qu'elle dût compter dans les conditions d'installation des moines. Au milieu du XII ${ }^{\mathrm{e}}$ siècle, l'ancienne cella carolingienne avait cependant végété et ne possédait plus qu'un rang de chapelle. Le monastère d'Aniane qui s'était imposé comme l'une des principales seigneuries entre les gorges de l'Hérault à l'ouest et l'ancienne agglomération du Roc de Pampelune à l'est, avait finalement concentré l'essentiel de ses intérêts au nord du site auprès d'une église paroissiale (Saint-Étienne de Viols) qui jalonnait une voie de communication permettant d'atteindre les Cévennes. Au sud de l'ancienne agglomération du $\mathrm{VI}^{\mathrm{e}}$ siècle, en revanche, de nouveaux pôles castraux (Murles, Vailhauquès, Montarnaud) s'étaient développés, tandis qu'à l'est les petits causses de garrigue étendus jusqu'à la ville nouvelle de Montpellier devaient connaître un destin singulier.

\section{Influence urbaine : Montpellier et le Bois de Valène ( $\mathrm{XIII}^{\mathrm{e}}-\mathrm{XVI}^{\mathrm{e}}$ siècles)}

Le locus de Saugras et l'ancienne agglomération du Roc de Pampelune semblent avoir constitué une sorte de borne marquant les limites orientales de la seigneurie anianaise, un espace de plusieurs milliers d'hectares qui s'étirait depuis le monastère sur une dizaine de kilomètres d'ouest en est et qui n'a jamais comporté de pôles castraux. Aux contacts méridionaux et orientaux du locus, les petits causses forestiers relevaient quant à eux des comtes de Substantion/Mauguio au cours des $\mathrm{XI}^{\mathrm{e}}$ et $\mathrm{XII}^{\mathrm{e}}$ siècles. Immédiatement à l'est du Roc de Pampelune et au nord du castrum de Murles, la documentation écrite individualise en tant que tel, et pour la première fois

7. L. SCHNEIDER, «Structures du peuplement et formes de l'habitat dans les campagnes du sud-est de la Gaule entre Antiquité et Moyen Âge (IV ${ }^{\mathrm{e}}-\mathrm{VIII}{ }^{\mathrm{e}} \mathrm{s}$.) : essai de synthèse », Gallia, 64, 2007 (à paraître). 


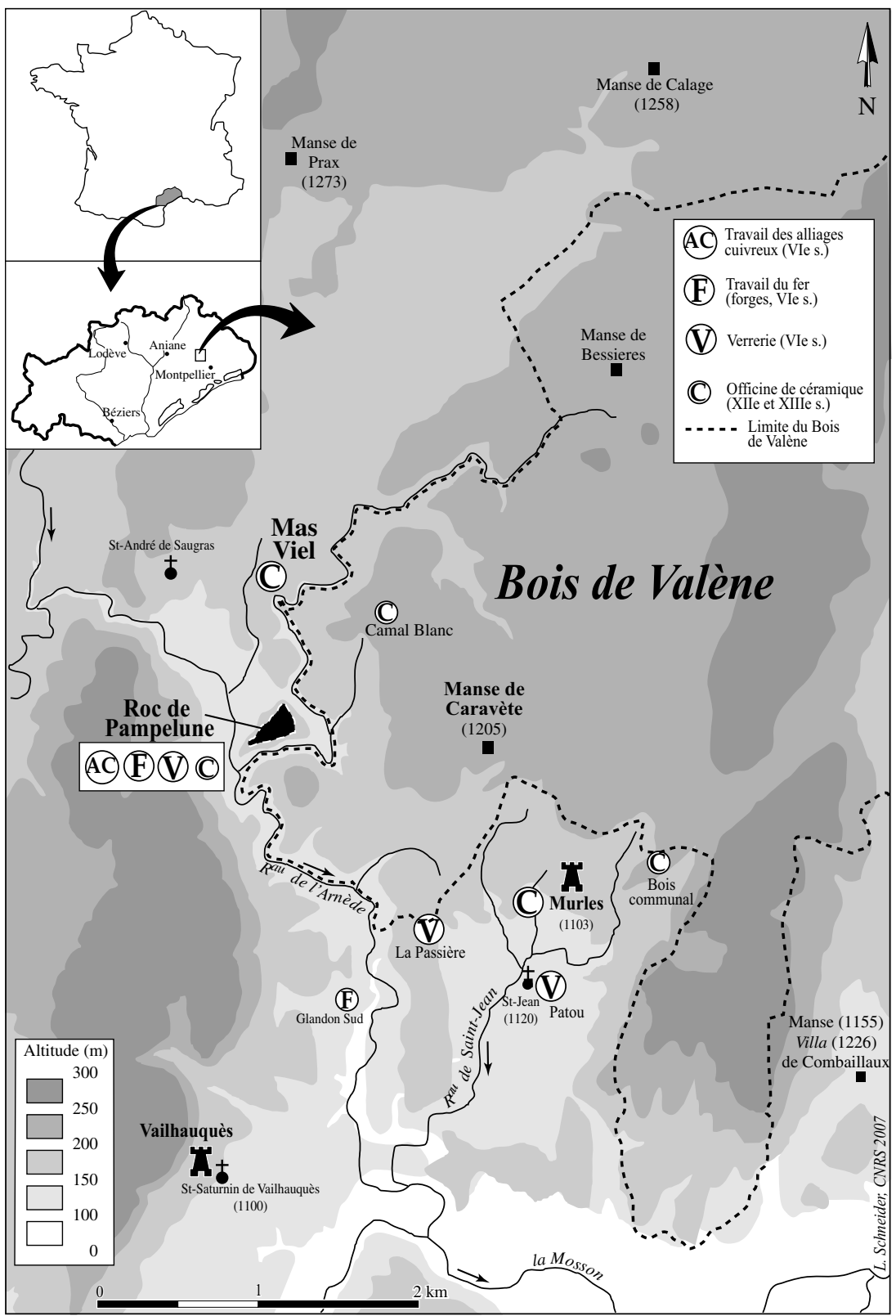


en 1189, un bois dénommé de Valène. Après la rétrocession du comté de Mauguio à l'évêché de Maguelone en 1215, celui-ci cède à son tour en acapte une part sur la frappe des deniers melgoriens et la totalité du «Bois de Valène » au tout jeune consulat de Montpellier contre 25000 sous et une albergue annuelle de 10 milites. La cession complète comprenait également tous les droits comtaux exercés sur le Bois ${ }^{8}$.

Évêques, consuls et communautés : la fixation du bois au XIII siècle

Fort de l'acquisition effectuée en 1215, le Consulat de Montpellier met rapidement en place sa juridiction sur ce territoire forestier et frappe aussitôt d'amendes et d'emprisonnement les sujets de l'évêque, habitants des manses et des paroisses du voisinage qui faisaient paître leur bétail dans le bois et continuaient d'exercer d'anciens droits de forestage. À partir de 1264, l'évêque de Maguelone attise les tensions et entame de longues procédures destinées à réclamer l'entière juridiction sur le bois. Un premier accord, fragile, partage le droit de justice entre la «basse justice» (permission de lever des amendes et autres punitions non mutilantes) accordée à Montpellier et la « haute justice» (emprisonnements, peine capitale) qui revient à Maguelone ${ }^{9}$. Cependant, de nouvelles disputes émergent et durent plus d'un siècle jusqu'en 1376. Pendant cette longue période de conflits, le Consulat de Montpellier et l'évêque de Maguelone s'affrontent notamment autour de la question d'une potence (furcis) installée au Bois de Valène par le Consulat, un instrument de justice que lui déniait le pouvoir épiscopal ${ }^{10}$. Cette seule affaire débouche, entre autres sanctions, sur l'excommunication de l'ensemble du Consulat en 1364 et 1369 , un recours à l'arbitrage du juge royal en 1371 , puis à celui du cardinal Grimoard en $1375^{11}$. Seul ce dernier arbitrage permet de mettre fin à la querelle et de revenir au statu quo de 1264 : partage équitable des justices entre les deux partis et rétablissement des «fourches de Valène », en échange d'une légère augmentation des albergues dues à l'évêque.

Si l'on songe que le Bois de Valène, une «garrigue » découpée par de profonds ravins et des massifs pierreux et accidentés n'atteignait même pas 3000 ha, ces longues querelles pourraient paraître bien incongrues. Le Bois de Valène constituait cependant pour la communauté de Montpellier une source de revenus qui était loin d'être négligeable. À certains moments de

8. J. Rouquette, A. Villemagne, Cartulaire de Maguelone, 6 vol., Montpellier, 19121927, I, p. 362, acte CCII [1189] et p. 144-150, acte CCCXLV [1215] ; Archives de la Ville de Montpellier (ci-après AVM), AA4-AA5, Grand Thalamus (microfilm 2 Mi 78), f ${ }^{\circ}$, acte 17, $\mathrm{f}^{\circ} 8$, acte 18 et $\mathrm{f}^{\circ} 26$, acte 46 .

9. J. Rouquette, A. Villemagne, op. cit., II, p. 50-54 [1264] ; AVM, Grand Thalamus, $\mathrm{f}^{\circ} 99$, acte 309 .

10. L.-J. Thomas, «La baronnie de Caravètes », Monspeliensia, 1-2, 1931, p. 87-157.

11. D'après AVM, Notice sur les anciens inventaires. Inventaire du Grand Chartrier, I, Montpellier, 1895-1899, cotes E7 2465, F6 2943 et 2932. 
l'histoire de la ville, dans les années vingt et trente du XvI siècle par exemple, la gestion du bois pouvait représenter en moyenne jusqu'à $23 \%$ des revenus de la ville et connaître des pics à $45 \%$, dépassant les tailles et autres impôts sur les biens urbains ${ }^{12}$. En effet, en vertu de son droit de basse justice, le Consulat de Montpellier se réservait le lucratif marché de l'affermage de coupes de bois et de pâturages, ainsi que le prélèvement des amendes aux contrevenants.

\section{Économie urbaine : la gestion d'un bois}

La gestion et les conditions d'exploitation de cette ressource forestière par le Consulat de Montpellier, une ville neuve qui ne disposait pas d'une grande assise foncière, peuvent être retracées de manière particulièrement détaillée par le biais des archives comptables ${ }^{13}$, des archives notariées ainsi que des statuts et règlements consulaires (Petit et Grand Thalamus). Source inédite, le Liber Recognicionum et Arrendamentorum nemoris Vallene, est un registre exhaustif, en trois tomes de plusieurs centaines de folios chacun, des affermages, ventes et autres cessions du XVI ${ }^{\mathrm{e}}$ siècle (de 1501 à 1584). Il constitue une source inégalée pour l'étude des rythmes d'exploitation du Bois ${ }^{14}$.

Dès les premiers comptes conservés (milieu du XIV ${ }^{\mathrm{e}}$ siècle), le Consulat met en place un système strict de contrôle de l'exploitation du Bois en taillis, régi par une division de l'espace en douze coupes nommées et bornées (tails) ${ }^{15}$. Pour contrôler l'exploitation, deux forestiers, parfois dénommés " escudiers de Valène », sont élus tous les quatre ans, et ont pour charge de surveiller le Bois, d'y assurer l'absence de chasse, de pâturages d'ovins et de locations illicites. Parallèlement, le Consulat baille à ferme sur la même durée l'exploitation de chaque coupe de bois et des pâturages à un ou plusieurs exploitants (rendiers) contre un loyer versé annuellement (variant de 171 à 2242 livres pour le XVI ${ }^{\mathrm{e}}$ siècle). En contrepartie, l'exploitant s'engage à veiller lui aussi sur le bois «durant lo temps de song arrendament et gardara et conservara lodit bosc a son poder comme se era sien propry ${ }^{16}{ }^{\prime}$, et surtout il a la charge de la fourniture en bois de chauffage (lenha), en bois d'œuvre pour les constructions publiques (fustas de Valena [...] quent ne

12. C. BRItTon, Du mas à la ville: contribution à l'étude de l'habitat dispersé en pays Montpelliérain (XII -XVI ${ }^{e}$ s.), Doctorat en cours, Université d'Aix-Marseille I. Calculs effectués d'après la documentation comptable citée ci-dessous conservée aux AVM (cotes CC 278, CC 531 à 572, CC 573 à 633, CC 634 à 663, CC 667 à 711, CC 712 à 756 (sauf 745, 747, 754), CC 757 à 843, CC 844 à 847) et archives notariées (cotes BB1 à 18).

13. AVM, Archives du Greffe de la maison consulaire XIV ${ }^{\mathrm{e}}-\mathrm{XVII}{ }^{\mathrm{e}}$ siècles : Livre des recettes de la Claverie 1357-1600.

14. AVM, DD 17, Liber Recognicionum et Arrendamentorum nemoris Vallene.

15. Les comptes consulaires conservent plusieurs actes notamment un prix-fait concernant l'achat, la taille et l'implantation des bornes de Valène entre 1482 et 1496 (AVM CC 563, $\mathrm{f}^{\mathrm{o}} 36, \mathrm{CC} 565, \mathrm{f}^{\mathrm{o}} 58, \mathrm{CC} 563, \mathrm{f}^{\mathrm{o}} 39$ et CC $578, \mathrm{f}^{\mathrm{o}} 31$ )

16. AVM, DD17, fo 29 (serment de 1505). 
auria hom besonh per los hospitals, molins, pontz et payssieyras del comu $)^{17}$ de la ville de Montpellier tout en suivant un cahier des charges extrêmement codifié quant aux modes d'exploitation. Ceux-ci sont minutieusement consignés dans les serments que doivent prononcer escudiers et rendiers lors de leur prise de fonction ${ }^{18}$.

En premier lieu, le Consulat rappelle que l'exploitant, comme les forestiers, a une obligation de garde constante du Bois contre la dépaissance des moutons et des chèvres et les coupes interdites. Le rendier doit également s'engager à exploiter en «bon père de famille » la ressource forestière : conserver de grands arbres à intervalles réguliers afin de délimiter l'étendue de la coupe de bois, réserver 100 grands arbres choisis par les consuls afin de fournir du bois de construction à la ville, interdire l'installation de fours à chaux sans l'accord des consuls, tailler respectueusement les arbres... Outre ces consignes, le serment insiste sur l'objectif principal de cette exploitation : fournir suffisamment de bois sec et mûr de qualité pour tous les habitants de la ville de Montpellier par l'exploitation de l'une des 12 (ou 13) coupes de bois. Le fermier du Bois de Valène doit également s'acquitter de la fourniture gracieuse de 100 quintaux de bois à la Maison du Consulat ainsi qu'un total de 134 quintaux aux hôpitaux de la Ville ${ }^{19}$. Cependant, l'exploitation n'est évidemment pas une opération charitable et le bois, destiné exclusivement à la ville de Montpellier sous peine de poursuites, est vendu sur place suivant une tarification et un gabarit contrôlés par le Consulat ${ }^{20}$.

En dépit de cette réglementation extrêmement stricte, le Bois de Valène reste une ressource en bois d'équilibre fragile. En 1407, lors d'une visite à Valène, les consuls le trouvent dans un état «si despolhat et entretriat de bons albres que non si poyrian a penas trubar VI o VII ${ }^{21} \gg$ qu'ils radicalisent d'autant plus les obligations des rendiers et escudiers. La même situation se répète un siècle plus tard, entre 1502 et 1510 , et à la suite de plusieurs infractions graves au règlement (ventes illégales de bois à des étrangers à la communauté, pâturages illicites de plus de 400 ovins, corruption du forestier, etc.), les Consuls vont jusqu'à faire installer des couchettes supplémentaires au Bois de Valène, afin de surveiller eux-mêmes les gardes du bois «lasquales fan mal leur dever ${ }^{22} »$.

17. M. Pégat, E. Thomas, C. Desmazes, Le Petit Thalamus, Quatrième partie. La Chronique Romane, Montpellier, 1838 p. 183-184.

18. Ces serments sont rédigés tous les quatre ans, avec certaines variantes, puis insérés dans les Thalamus, puis dans le Liber Recognicionum et Arrendamentorum nemoris Vallene.

19. Tous ces éléments proviennent du Liber Recognicionum et Arrendamentorum nemoris Vallene, AVM, DD DD17.

20. En 1407 : les tarifs sont de : 15 deniers la saumée de «grosse bête bien chargée », 10 deniers la saumée d'animal plus petit que l'âne, 2 sous 6 deniers la charretée ; et les dimensions minimales réglementaires sont: «per lo balous (fagots ?) » 4 pans et demi de longueur, pour le bois de construction, 5 pans.

21. M. Pégat, E. Thomas, C. Desmazes, Le Petit Thalamus, op. cit., p. 171.

22. «Livre de mémoyres" (1508, $\left.\mathrm{f}^{\circ} 53\right)$, Brèves des réunions consulaires (1491-1519) dans M. Oudot de Dainville, Archives de la ville de Montpellier. Inventaires et documents, XI : Documents comptables, Montpellier, 1959, p. 72-96. 
Ce tableau rapide des règlements concernant le Bois de Valène à l'époque médiévale et moderne, démontre l'importance économique que constituait cette ressource en bois pour la ville. Cependant, la volonté d'autosubsistance de Montpellier de chauffage et d'œuvre contraste singulièrement avec les listes de leudes conservées pour le milieu du XIV ${ }^{\mathrm{e}}$ siècle ${ }^{23}$. À cette époque, en effet, le Consulat perçoit un montant sur «cascuna saumada de lenha que vengua foras lo termenal de Montpelier », les pommes de pin pour les fours et sur la fuste de Quilhan.

\section{Autres ressources...}

Outre l'approvisionnement urbain en fuste et en lenha, les fonds dépouillés permettent aussi de souligner l'exploitation directe ou indirecte par le Consulat d'autres productions comme la chaux. On note ainsi quatre mentions de constructions et d'utilisations de fours à chaux entre 1357 à 1552. Prérogative seigneuriale, semble t-il, le Consulat est la seule autorité à pouvoir accorder le droit d'installer de tels outils de production dans le Bois de Valène. Ce droit peut également être cédé, comme on l'observe autour de Mireval lorsque le seigneur aliène «potestatem et licentiam faciendi furnum, vel furnos caucerencs, vel de lignis facere tallare et percipere, vel quicquid ibi facere volueritis, sicut in tuo proprio honore ${ }^{24} »$. Dans ces circonstances, le Consulat accorde en 1367 à Raimond de Montlaur, seigneur de Murles - seul cas connu des archives -, la licence de créer un four à chaux, pour son propre compte et sans avoir droit d'utiliser le bois provenant de Valène. Dans la plupart des cas, en revanche, il désigne directement les forestiers et caussyniers qui vont produire de la chaux pour l'usage de la ville ${ }^{25}$. Malgré le faible nombre de mentions de fours à chaux consulaires dans le Bois de Valène, un exemple particulièrement frappant de leur ampleur apparaît dès $1357^{26}$. À cette date, le Consulat emploie plus de 1105 forestiers (en jours/ homme) et un maître-chaufournier pendant sept mois (payé 273 livres) pour alimenter un four. On ne connaît malheureusement pas la destination de la chaux produite mais on peut supposer qu'elle a servi à des constructions montpelliéraines.

23. Cet acte, coté AMV CC 844 (Commandement général 1356-1371) a été égaré et subsiste seulement sous forme d'inventaire dans M. Oudot de Dainville, Archives de la ville de Montpellier. Inventaires et documents, IX : Archives du Greffe de la maison consulaire, Montpellier, 1949, p. 177.

24. A. Germain, C. ChabANneAu éd., Liber instrumentorum memoralium ou cartulaire des Guilhems de Montpellier, Montpellier, 1884-1886, p. 535, acte CCCXLIII [1157].

25. Par exemple, en 1535 les consuls paient 10 livres à Jean Coste de Murles pour une cremade qui a fourni le jardin de la Madeleine à Montpellier (AVM, CC 744, $\mathrm{f}^{\circ}$ 8), et en 1522 acompte fait directement par le Consulat à Pierre Romenières et Girard Alegres, caussyniers $\left(\mathrm{AVM}, \mathrm{CC} 754, \mathrm{f}^{\circ} 55 \mathrm{v}^{\circ}\right)$.

26. Cet acte, coté AVM, CC 845 (Commandement général 1356-1371), fo 80, a été égaré et subsiste seulement sous forme d'inventaire dans M. Oudot de DaInviLle, Archives de la ville de Montpellier. Inventaires et documents, op. cit., p. 179. 
En dehors de ces quelques mentions de fours à chaux, d'autres productions attendues comme celle du tan, de la poix ou de charbonnières, ne transparaissent guère dans la documentation écrite, à l'exception de leur présence dans les lauzimes de la ville de $1356^{27}$. Liste des tarifs appliqués sur les matières premières entrant en ville, elle explicite l'origine étrangère à la communauté des matériaux lorsque cela est nécessaire ("que venguon de foras, que venga foras, etc. »), ce qui tend à suggérer l'origine locale des autres produits. Ainsi défilent des saumées de branchages, de charbon de pierre ou de bois, de chaux, de plâtre, cargaisons de tan, sacs de rodoul (Coraria myrtifolia), mais également toute une liste d'objets façonnés en bois : planches, lattes et merrains, bois d'œuvre non scié, divers tonneaux, cuves, cercles de tonneaux et de cuves, divers piquets, pieux, fourches, bâtons, bâts, râteaux, poteaux, éléments d'enclos, pieds de meubles, hottes et comportes pour la vendange, divers objets pour la vinification et enfin vaisselle en bois.

\section{L'apport de l'archéologie: une production originale de céramique associée au marché montpelliérain?}

Les programmes de prospections, de sondages et de fouilles conduits autour du Roc de Pampelune et du Bois de Valène mettent encore l'accent sur le développement d'activités artisanales non enregistrées par les sources écrites. C'est le cas notamment de l'activité potière. Dans un rayon n'excédant pas deux kilomètres autour du Mas de Caravète, centre de gestion du Bois de Valène, ont été repérés deux grands ateliers de production potière et trois officines secondaires qui paraissent peu ou prou avoir fonctionné simultanément au moins durant les deux premiers tiers du XIII ${ }^{\mathrm{e}}$ siècle. La concentration de tels ateliers dans un si petit secteur est remarquable, unique sans doute à l'heure actuelle en milieu de garrigue. L'un des plus grands ateliers, celui de Mas-Viel (Argelliers, Hérault) a pu bénéficier d'un premier sondage qui a révélé les caractères originaux d'un répertoire de formes produites en mode oxydant, trait innovant qui rompt avec une tradition séculaire des vases gris et noirs hérités du haut Moyen $\mathrm{Age}^{28}$. Cette céramique, abondamment diffusée dans l'arrière-pays montpelliérain, est également bien représentée sur la table montpelliéraine comme l'attestent désormais diverses fouilles urbaines. Malgré une datation obtenue par radiocarbone sur la dernière cuisson du seul four fouillé [C14 $765 \pm 30$ BP calibré 1220-1287 AC], on ne peut être assuré que le développement de cet artisanat soit directement lié à l'action des consuls de Montpellier. Il semble néanmoins que c'est au cours du XIII ${ }^{\mathrm{e}}$ siècle que cette activité a atteint son acmé. La localisation de ces ateliers, sur des reliefs rocheux, en milieu de garrigue pouvait paraître

27. AVM, CC 844, voir ci-dessus.

28. H. Breichner, L. Chabal, L. Lecuyer et L. Schneider, « Artisanat potier et exploitation du bois dans les chênaies du nord de Montpellier au XIII ${ }^{\mathrm{e}}$ siècle (Hérault, Argelliers, Mas-Viel) », Archéologie du Midi Médiéval, 20, 2002, p. 57-106. 
insolite. Elle prend tout son sens cependant si l'on mesure l'arrière-plan des activités intensives sous-tendues par l'exploitation et la gestion du Bois de Valène, qui plus est dans la perspective d'un débouché urbain. L'affermage des coupes pouvait d'ailleurs participer au développement de cette production.

L'analyse anthracologique des combustibles découverts dans le four $1 \mathrm{de}$ Mas-Viel, et les parallèles qui peuvent être faits avec les données préliminaires du Roc de Pampelune permettent d'extrapoler une modification du paysage entre la fin de l'Antiquité et le XIII ${ }^{\mathrm{e}}$ siècle. Les taillis assez matures du $\mathrm{VI}^{\mathrm{e}}$ siècle, riches en Chêne blanc et en Chêne vert, ont cédé la place au XIII ${ }^{\mathrm{e}}$ siècle, sous l'effet des coupes répétées, à des taillis de Chêne vert dominant, riches en Buis et autres arbustes. Le calibre et l'âge des bois utilisés comme combustible dans le four 1 de Mas Vieil montrent en outre de manière très concrète une gestion des coupes raisonnée, par rotations de 10-12 ans et révolutions de 30-36 ans, permettant l'obtention de fagots calibrés, impératif technique qui conditionne l'art de la conduite du feu au moment des cuissons des céramiques. Ce type d'approche qui met au jour les pratiques réelles des artisans éclaire d'une autre manière l'existence d'une gestion raisonnée de cet espace forestier. Le développement d'un artisanat potier au XIII ${ }^{\mathrm{e}}$ siècle paraît avoir été sous-tendu par la mise en place d'une économie de fagotage, sous-produit de taillis gérés par ailleurs pour du bois de plus fort calibre.

Les petits causses de l'arrière-pays montpelliérain ont longtemps été perçus comme des espaces sans histoire, immobiles, déserts et finalement peu exploités avant les conquêtes médiévales des $\mathrm{XI}^{\mathrm{e}}-\mathrm{XII}^{\mathrm{e}}$ siècles et le développement du système des «mas ». Quelques épaves documentaires les désignaient tout au plus comme des terrains de parcours privilégiés pour le bétail à partir de la fin du VIII ${ }^{\mathrm{e}}$ siècle et au cours du $\mathrm{IX}^{\mathrm{e}}$ siècle, dans le cadre de l'éclosion monastique que connut ce secteur. La faiblesse du maillage castral laissait elle-même supposer que la colonisation des $\mathrm{XI}^{\mathrm{e}}-\mathrm{XII}^{\mathrm{e}}$ siècles avait été largement incomplète, même si l'élevage y avait été plus ample et plus spécialisé $^{29}$. L'enquête conduite autour de l'ancien locus carolingien de Saugras et du Bois de Valène, soit sur un espace réduit à moins de 5000 ha, montre combien l'histoire de ces espaces est pourtant complexe, nuancée, faite d'héritages et de traits de développement spécifiques difficilement généralisables d'un plateau ou d'un causse à un autre. Elle est surtout beaucoup plus riche qu'on ne pouvait l'imaginer, tant la production de données exige aujourd'hui un labeur infini et surtout des pratiques d'interdisciplinarité qui seules permettent de sonder ce monde rural dans ses activités les plus concrètes. Pour les périodes historiques, la première empreinte laissée par l'homme dans ce secteur est celle des années 450-550. La période est marquée par l'installation soudaine d'un centre de peuplement développant une économie forestière et artisanale bien structurée. Le travail du fer, des métaux cuivreux et du verre n'est pourtant, dans la culture matérielle, que l'aspect le plus visible

29. A. Durand, Les paysages médiévaux du Languedoc ( $X^{e}-$ XII $^{e}$ s.), Toulouse, 1998. 
d'une activité économique sans doute plus encore polyvalente mais globalement orientée autour des ressources naturelles au titre desquelles, le bois, sous toutes ses formes, trouve une place importante. L'identification de verreries forestières à cette date haute constitue un trait original qu'il convient de souligner car pour l'heure sans équivalent dans le midi de la Gaule. Après un siècle d'occupation, l'abandon de l'agglomération relâche plusieurs siècles durant la pression qui avait été initiée sur le milieu sans que l'on saisisse comment se sont effectués la redistribution du peuplement ou le transfert de population. L'installation d'une cella monastique marque néanmoins l'ancrage de nouveaux pouvoirs à la fin du VIII ${ }^{\mathrm{e}}$ siècle. Elle constitue un phénomène de résilience qui donne une nouvelle personnalité juridique à un espace (re)devenu territoire, sans que celui-ci ne retrouve cependant la pression anthropique connue à la fin de l'Antiquité. À partir des $\mathrm{XI}^{\mathrm{e}}-\mathrm{XII}^{\mathrm{e}}$ siècles la multiplication des mentions de manses témoigne de l'intérêt persistant porté à ces espaces par l'abbaye d'Aniane mais aussi de nouvelles variations et d'une occupation de plus en plus dense, quoique relative. Des mentions éparses de forges suggèrent l'existence de pratiques métallurgiques, mais là encore sans jamais atteindre le niveau connu autour des années 500. Dans ces terres monastiques et comtales, peu touchées par le dynamisme castral, c'est en définitive l'émergence du pôle urbain montpelliérain qui a contribué, au moins depuis le seuil du XIII ${ }^{\mathrm{e}}$ siècle, à désenclaver ces espaces désormais situés à moins d'une vingtaine de kilomètres d'une ville nouvelle. On a souvent associé Montpellier à la mer et à l'espace littoral. Le dossier du Bois de Valène, rapidement présenté ici - curieusement longtemps demeuré inexploité malgré la richesse du fonds conservé -, montre combien l'arrière-pays forestier a compté lui aussi dans l'économie de la ville, notamment aux premiers temps du Consulat. Le développement spectaculaire d'officines de potiers, dont la production innovante (mode de cuisson oxydant) est abondamment diffusée vers la ville, est un autre témoignage de la vitalité de ces espaces et de la diversité des nouvelles expériences qui ont été conduites entre la seconde moitié du XII ${ }^{\mathrm{e}}$ siècle et le second tiers du XIII ${ }^{\mathrm{e}}$ siècle. L'exemple vaut aussi et surtout parce qu'il nous renseigne sur une activité que n'enregistrent pas les sources écrites et ce malgré la richesse du fonds conservé. Un exemple parmi combien d'autres qui ne se laissent guère aborder tant les traces documentaires ou matérielles qu'ils ont laissées demeurent discrètes et sans doute à bien des égards imperceptibles?

Charlotte BRITTON, Doctorante, allocataire de recherche, LAMM, UMR 6572, 5 rue du Château de l'Horloge, BP 647, F-13094 Aix-en-Provence Cedex 2 - Charlbritton@aol.com

Lucie Chabal, CNRS, Centre de Bio-archéologie et d'écologie, UMR 5059, CNRS-Université de Montpellier I, Institut de Botanique, 163 rue A. Broussonet, F-34090 Montpellier - chabal@univ-montp2.fr

Gaspard PAGÈs, Doctorant, allocataire de recherche, UMR 5140 CDAR, 390 avenue de Pérols, F-34970 Lattes - gaspard.pages@free.fr 
Laurent SchNEIDER, CNRS, LAMM, UMR 6572, 5 rue du Château de l'Horloge, BP 647, F-13094 Aix-en-Provence Cedex 2 - lauschne@club-internet.fr

Approche interdisciplinaire d'un bois méditerranéen entre la fin de l'antiquité et la fin du Moyen Âge, Saugras et Aniane, Valène et Montpellier

Le développement économique que connaît la ville de Montpellier au $\mathrm{XIII}^{\mathrm{e}}$ siècle a souvent été associé à celui de l'espace littoral. Pourtant l'influence de la nouvelle métropole s'étend aussi dans son proche arrière-pays. $\mathrm{Au}$ seuil du XIII ${ }^{\mathrm{e}}$ siècle les consuls ont exploité les ressources forestières d'un espace de garrigue identifié sous le nom de «Bois de Valène ». Une récente enquête collective permet désormais de mieux saisir l'histoire de cet espace dans le temps long. La garrigue montpelliéraine n'était pas seulement vouée au pastoralisme. Entre le $\mathrm{v}^{\mathrm{e}}$ siècle de notre ère et le $\mathrm{XVI}^{\mathrm{e}}$ siècle, les secteurs du Bois de Valène et de Saugras ont été en effet exploités de manière multiforme. Métallurgie du fer, charbonnage, verreries rurales et officines de céramiques notamment montrent que ces zones dites de marge n'étaient pas des espaces immobiles. Découvertes archéologiques, textes et analyses de laboratoire offrent aujourd'hui des lectures croisées à même d'éclairer une partie de ces realia dont les traces demeurent le plus souvent bien fugaces.

Ressources forestières - métallurgie - ateliers de céramique - Bois de Valène - temps long - garrigue montpelliéraine - arrière-pays

An interdisciplinary approach of a Mediterranean wood between the end of Antiquity and the end of the Middle Ages. Saugras and Aniane, Valène and Montpellier

The economic development which happened in the town of Montpellier in the 13th century has often been associated with the development of its coastal area. Yet the influence of the new metropolis extends into its nearby hinterland. At the beginning of the 13th century the consuls of Montpellier exploited the forest resources of an area of Mediterranean scrubland named the « Bois de Valène ». A recent collective study now lets us into the history of this area through a long time-span. The scrubland around Montpellier wasn't just restricted to pastoral uses. Between the 5th and 16th centuries A.D., the areas around the «Bois de Valène » and Saugras were exploited in many different ways. Ironworks, coal furnaces, rural glassworks and pottery kilns amongst others show that these areas, considered as marginal, weren't motionless spaces. Archaeological discoveries, written records and laboratory analyses now offer cross-readings which let light into a part of these realia, more often than not known through extremely fleeting artefacts.

Forest materials - metal-work - pottery kilns - Bois de Valène - long timespan - Montpellier scrubland - hinterland 